\title{
El capital intelectual en el clúster de cuero y calzado de la Provincia de Tungurahua
}

\section{The intellectual capital in the leather and footwear cluster of the Province of Tungurahua}

\author{
Juan Carlos Erazo Álvarez ${ }^{1 *}$ \\ ${ }^{1}$ Universidad Católica de Cuenca \\ *jcerazo@ucacue.edu.ec
}

DOI: https://doi.org/10.26871/killkana_social.v2i3.333

\begin{abstract}
Resumen
El Capital Intelectual es el conjunto de aportaciones no materiales que en la era de la información se entienden como uno de los principales activos de las empresas. En las micro, pequeñas y medianas empresas se necesita identificar elementos intangibles que puedan generar valor. La investigación realizada identificó como problema científico la necesidad de caracterizar el capital intelectual en las micro, pequeños y medianos empresas del clúster de cuero y calzado de la Provincia de Tungurahua, se propuso como objetivo identificar factores de éxito, lo que puede contribuir a diseñar una metodología para la gestión del capital intelectual en el mencionado clúster. Se concluye que la caracterización realizada, permitió conocer las principales fortalezas y debilidades y posibles áreas de mejora, la integración realizada de la actividad en los negocios de cuero y calzado a las áreas de enfoque del capital intelectual, permitió identificar los elementos que tienen posibilidades de generar valor para la organización.
\end{abstract}

Palabras clave: capital intelectual, clúster.

\begin{abstract}
The intellectual capital is the set of intangible contributions that becomes one of the most important companies assets into the information age. It is necessary to identify the intangible elements that can create value in micro, small and medium-sized enterprises. This research identified as scientific problem the need to characterize the intellectual capital in micro, small and medium-sized enterprises of the leather and footwear clusters in Tungurahua. The main goal was to identify elements of success that contribute to design a kind of methodology for the management of intellectual capital in the aforementioned clusters. It concludes that the characterization of intellectual capital allowed to realize different aspects: the recognition of the main strengths, the weaknesses, and the possible areas of improvement; the integration obtained from the business activity in the leather and footwear fields to the focus areas of intellectual capital; and the identification of elements that generate value for the organization.
\end{abstract}

Key words: intellectual capital, cluster.

\section{Introducción}

La presente investigación identificó como problema científico la necesidad de caracterizar el capital intelectual en las micro, pequeñas y medianas empresas del clúster de cuero y calzado de la Provincia de Tungurahua, se propuso como objetivo identificar factores de éxito, agrupados en las cinco áreas de enfoque que propone el modelo de navegador de Skandia (Edvinsson, 2007), lo que puede contribuir a diseñar una metodología para la gestión del capital intelectual en el mencionado clúster.

\section{Marco teórico o antecedentes}

Según (Ruso, 2011), la utilidad que se genere de una mercancía, es lo que se define como valor de uso que no sólo se representa en términos monetarios y no es solamente producto de un acto de compraventa, es la cualidad más significativa de los intangibles.

Para (Nevado y López, 2002) los activos intangibles tienen naturaleza inmaterial, sin sustancia o esencia física, así como poseen capacidad para generar beneficios económicos futuros que pueden ser controlados, como un bien que puede generar futuras riquezas. (Borras, 2014), (Nevado y López, 2002). 
Los activos intangibles, vinculados al conocimiento y su combinación holística, ejercen una gran influencia para alcanzar los objetivos estratégicos de una empresa, no es suficiente el soporte material y financiero, es imprescindible contar con los recursos intangibles. (Borrás, Ruso, y Campos, 2011), (Borras, 2014).

Los pioneros en la conceptualización y medición del capital intelectual (Edvinsson, 2007), (Stewart, 2015), (EUROFORUM, 1998) refieren dos grandes clasificaciones del capital intelectual: capital humano, que incluye conocimientos, habilidades, actitudes, y destrezas de las personas que componen las organizaciones y capital estructural que contiene los conocimientos desarrollados y explicitados por las organizaciones, integrado por el capital clientes, procesos y capacidad de innovación.

Según (Rivero, 2009), (Monagas, 2012), el capital intelectual es un conjunto de activos intangibles y aportaciones no materiales, es todo lo que ha conseguido en términos de relaciones, procesos, descubrimientos, innovaciones, sistemas de formación, presencia en el mercado e influencia y reconocimiento en la sociedad en una organización.

Se considera además una herramienta del desarrollo competitivo, una técnica para mejorar el posicionamiento y se relaciona con los procesos comunicacionales que facilitan la construcción de una memoria empresarial. (Contreras, Díaz, y Hernández, 2012)

Para (Simó y Sallán, 2008) El capital intelectual es el conocimiento propiedad de la organización (conocimiento explícito) o de sus miembros (conocimiento tácito) que crea o produce valor presente para la organización.

Según (Betancur, 2015) el concepto tiene dos perspectivas, la contable y la estratégica de la generación de valor, para (Stanimirov, 2014) el compromiso en actividades de responsabilidad social influye positivamente en el capital intelectual, ya que establece una cultura basada en la confianza y la preservación del entorno, (C. G. J., Salazar-Mesa, L., 2013) considera que es una limitación del concepto no tomar en consideración intangibles no intelectuales, tales como valores y cultura organizacional.

Exponen (Alama, 2008), (Aceves, 2010) que los recursos intangibles y capacidades (capital intelectual) se comprenden mejor dentro del contexto específico en el que se desarrollan y son fuente importante para conseguir la ventaja competitiva, aunque (Valencia, 2005) especifica que no todos los conocimientos que la organización tiene, serán fuentes de ventajas competitivas sostenibles sino solamente aquellos que contribuyan decisivamente a la generación de valor económico

Se considera además una herramienta del desarrollo competitivo, una técnica para mejorar el posicionamiento y se relaciona con los procesos comunicacionales que facilitan la construcción de una memoria empresarial. (Contreras y cols., 2012)

El conocimiento del valor de los activos intangibles .${ }^{\circ}$ cultos. ${ }^{\circ}$ capital intelectual que tiene una economía se convierte en elemento clave para estimar el "valor real"de la misma, ya que, no es suficiente con la cuantificación del valor de producción para estimar las diferencias en la economía del conocimiento, puesto que, se requiere, además, una valoración adecuada de la innovación y el factor humano.

\section{Metodología}

La estrategia de la investigación siguió el orden siguiente:

1) Reunión con dirigentes gremiales datos del clúster de cuero y calzado, de la Provincia de Tungurahua, para valorar el formulario de preguntas a realizar.

2) Se elaboraron rutas para el levantamiento de información, de acuerdo a la base de datos del clúster de cuero y calzado.

3) Se aplicó el cuestionario rediseñado a 359 propietarios de negocios de cuero y calzado

4) Se integraron los resultados en las cinco áreas de enfoque que propone el modelo de navegador de Skandia (Edvinsson, 2007)

\section{Resultados}

Para el desarrollo de la investigación se tuvo en cuenta el criterio en cinco enfoques (Edvinsson, 2007), en las cuales su mayor medición es la generación de valor analizando básicamente los activos intangibles, los cuales son: financiera, clientes, personas, procesos, renovación y desarrollo.

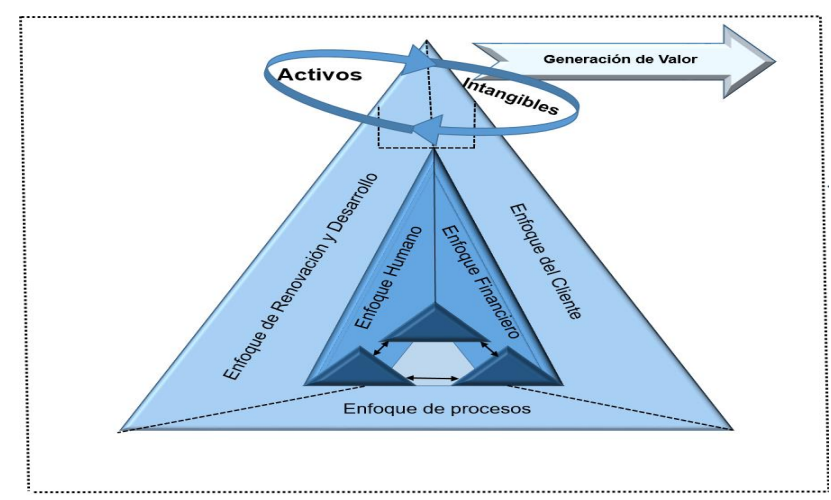

Figura 1.

\subsection{Enfoque Financiero}

Este aspecto se relaciona con el conocimiento y análisis de los aspectos financieros como la rentabilidad y el crecimiento.

En el caso del clúster objeto de análisis se identifica la necesidad de desarrollar los elementos que generen valor siguiente:

- El registro de sus cuentas de ingresos o gastos por medio de la contabilidad.

- Y el manejo de la rotación de inventarios 
En los resultados del cuestionario aplicado se aprecia que el $45 \%$ de los negocios no registra sus cuentas por medio de la contabilidad y el $49 \%$ no maneja la rotación de inventarios.

La utilización de la contabilidad es necesaria para administrar de la mejor manera posible los recursos financieros, ya que por medio de ella se obtiene una información útil para la toma de las decisiones económicas, se profundiza en el control de la gestión, se decide la realización de inversiones.

\subsection{Enfoque del cliente}

Se refiere a la habilidad del negocio para identificar las necesidades de los clientes.

En el caso del clúster objeto de análisis se identifica la necesidad de desarrollar los elementos que generen valor en los elementos siguientes:

- La importancia del estudio de mercado previo para diseñar el manejo de la imagen del producto.

- El desarrollo de los planes de marketing

- La estrategia de promoción utilizada

- Los valores corporativos que brinda la empresa al cliente

- La importancia del estudio de mercado para diseñar el manejo de la imagen del producto.

- El manejo de los planes de marketing para el lanzamiento de sus productos.

- Los canales o medios de comercialización utilizados.

El $56 \%$ considera la necesidad de un estudio de mercado previo para diseñar el manejo de la imagen del producto, sin embargo, el $60 \%$ no maneja planes de marketing para el diseño de los productos

Más del $66 \%$ utiliza su propio punto de venta y los distribuidores minoristas para vender sus productos, solo un $20 \%$ es a empresas comercializadores, mayoristas y cadenas de ropa.

Solo el $25 \%$ realiza acciones relacionadas con una estrategia de promoción, estas se caracterizan por afiches, utilización de la televisión, la radio e internet.

Los valores corporativos que brindan estos negocios a los clientes son precio justo, calidad, eficiencia, respeto, originalidad, comodidad, durabilidad.

La administración de relaciones con los clientes genera un elevado valor para estas organizaciones, el valor constituye la valoración objetiva que hace el cliente sobre la utilidad de una oferta en función de los beneficios que percibe respecto de sus costos, en este sentido pueden ser elementos la calidad, el precio y la conveniencia.

Otro elemento a analizar es la marca, como valoración subjetiva e intangible del consumidor respecto de la marca, el conocimiento de esta, por parte de los consumidores, su actitud y la percepción ética de la misma, se aprecia un insuficiente trabajo con el objetivo de posicionar una marca en los productos ofertados por estos negocios.

En las relaciones, es importante valorar los programas de lealtad, los de reconocimiento y de creación de conocimiento, en este caso las relaciones personales son fundamentales para la negociación con los proveedores y clientes

\subsection{Enfoque de Procesos}

Se analizan los procesos para crear servicios y productos para los clientes.

En el caso del clúster objeto de análisis se identifica la necesidad de desarrollar los elementos que generen valor en los elementos siguientes:

- Tipo de calzado.

- Pares de zapatos elaborados por semana

- Certificaciones que tiene o están en proceso.

- Procesos de seguridad industrial

- La unidad de planificación de producción.

- El proceso de producción

- El control de la calidad.

- Quien realiza control de calidad

- Cómo se realiza el proceso de corte

- La elaboración de las colecciones completas de calzado.

- La elaboración por parte del negocio de las colecciones.

- Las actividades realizadas en el proceso de diseño y producción.

- La realización de los modelados de sus diseños

- La elaboración de los prototipos.

- El proceso de armado y acabado final del calzado.

- La existencia de un departamento de ventas.

- La elaboración de un plan de contingencia de riesgos laborales

- La elaboración de un sistema de gestión de calidad.

- La aplicación de un proceso de mejoramiento continuo.

- El manejo de las normativas de trabajo.

- La realización de servicio de post venta.

- La planificación de las ventas.

- La recepción de asesoría o asistencia técnica

- La influencia de la presentación del producto en las ventas

Los resultados indican que el $73 \%$ de estos negocios fabrica zapatos para caballero y dama, con mayor número para damas, otras líneas de producción se relacionan con los zapatos de niño, deportivo, botas, etc., más del $60 \%$ elabora entre 51 y 200 pares de zapatos por semana.

El $48 \%$ no tiene certificaciones en proceso y el $43 \%$ no aplica procesos de seguridad industrial. El $42 \%$ de la producción se realiza en línea y el $31 \%$ por lote, aunque el $45 \%$ de los negocios no cuentan con una unidad de planificación.

El control de la calidad se realiza en igual medida por producto y por proceso y en el $57 \%$ es realizado por el gerente.

El $73 \%$ realiza el proceso de corte manual, el $32 \%$ elabora colecciones completas de calzado, el $31 \%$ son elaboradas por el negocio. 
El $42 \%$ elabora los diseños teniendo en cuenta revistas y catálogos y el $24 \%$ son copia de productos ya existentes, el $38 \%$ elabora los modelados de los diseños. El $44 \%$ de los negocios, antes de la producción del diseño, elabora un prototipo y el $51 \%$ el proceso de armado y acabado final del calzado lo hace en su planta.

Más del $50 \%$ no elaboran un plan de contingencia de riesgos laborales, aunque se observa un $34 \%$ que cuentan o están confeccionando un sistema de gestión de calidad y un $43 \%$, aplican algún proceso de mejoramiento continuo. El $45 \%$ no maneja normativas de trabajo y el $43 \%$ no recibe asesoría.

Más de la mitad de estas organizaciones no tienen departamento de ventas, el $54 \%$ no realiza servicio postventa y el 46 no planifica las ventas, aunque el $58 \%$ considera que la presentación del producto influye en sus ventas.

(Alonso, 2014) expone los beneficios del uso del enfoque de procesos.

1) Permite medir la actuación de la organización, reducir los costos internos innecesarios (actividades sin valor agregado) y acortar los plazos de entrega (reducir tiempos del ciclo) para mejorar la calidad de los productos/servicios.

2) Revela los procesos relacionados con los factores críticos para el éxito y los que son redundantes e improductivos.

3) Define el grado de satisfacción del cliente interno o externo y lo compara con la evaluación del desempeño personal.

4) Identifica las necesidades de los usuarios o clientes externos y orienta a la organización para compatibilizar la mejora de la satisfacción del cliente con mejores resultados organizacionales.

5) Permite entender las diferencias de alcance entre la mejora orientada a los procesos (qué y para quién se hacen las cosas) y aquella dirigida a los departamentos o a las funciones (cómo se hace).

6) Revela aquello que es positivo del trabajo en equipo contra el trabajo individual y la eficacia de los procesos con una óptica integradora en lugar de parcial.

7) Apunta a la organización en torno a resultados y no a tareas.

8) Mantiene los procesos bajo control, mejora continuamente su funcionamiento global y reduce su inestabilidad a causa de cambios imprevistos.

Se observa en las organizaciones objeto de estudio la necesidad de mejorar el funcionamiento interno de sus negocios en función de perfeccionar sus procesos de manera tal que generen valor para sus clientes.

\subsection{Enfoque de renovación y del desarrollo}

Se analizan las acciones a desarrollar para garantizar el crecimiento y la rentabilidad.

En el caso del clúster objeto de análisis se identifica la necesidad de desarrollar los elementos que generen valor en los elementos siguientes:
- $\quad$ La creación de una unidad de diseño.

- La investigación creativa para el desarrollo del calzado

- El análisis de tendencia para el desarrollo del calzado.

- El manejo de planes de marketing para el lanzamiento de sus productos

- El conocimiento de la competencia

- La creación de un departamento de innovación para el diseño de sus productos

- El tiempo estimado para innovar el diseño de sus productos

- La creación de alianzas con otras empresas.

- La participación en misiones empresariales fuera del país.

- La participación en ferias especializadas fuera del país.

- La protección de los diseños exclusivos

- Las oportunidades que pueden favorecer el negocio

- La innovación de los productos

Los resultados indican que el $60 \%$ no cuentan con unidad de diseño, sin embargo más del $40 \%$ realiza investigación creativa y análisis de tendencias para desarrollo del calzado. Más del $50 \%$ conoce su competencia, sin embargo no manejan planes de marketing para el lanzamiento de sus productos

El $58 \%$ no cuenta con un departamento de innovación para el diseño de sus productos, no obstante más de la mitad de los negocios consideran que es prudente semestralmente innovar el diseño de sus productos.

El $46 \%$ está dispuesto a realizar alianzas con otras empresas, a pesar de que solo el $11 \%$ ha tenido la oportunidad de participar en misiones empresariales fuera del país y el $8 \%$ en ferias especializadas.

El $26 \%$ utiliza la marca para proteger sus diseños exclusivos, el $21 \%$ con patentes y el $17 \%$ mediante un ambiente confortable.

Se aprecia que se pueden favorecer los negocios mediante la variedad de los modelos $(40 \%)$, el reconocimiento a nivel nacional (31\%), la diversidad en colores $(17 \%)$ y la alternativa de precios $(21 \%)$.

Estas organizaciones innovan productos teniendo en cuenta la asistencia a ferias (34\%), los modelos en revistas $(28 \%)$, los modelos en internet $(27 \%)$ y los catálogos de temporada $(21 \%)$.

Como se observa aun quedan muchos espacios por emplear en estas organizaciones que generen valor en función de desarrollar el negocio.

\subsection{Enfoque Humano}

El objetivo fundamental es generar el mejor ambiente laboral para los empleados y colaboradores, donde estos se encuentres satisfechos para que así mismo generen resultados óptimos.

En el caso del clúster objeto de análisis se identifica la necesidad de desarrollar los elementos que generen valor en los elementos siguientes:

- Aplica procesos de higiene personal

- La planeación de los recursos humanos 
- Atención a problemas de presión en el trabajo, discriminación y acoso sexual

- El análisis de puestos

- Procesos de capacitación para el personal

- ¿En qué temas aplica los procesos de capacitación?

- La evaluación de desempeño.

- Sitio web para reclutar personal en línea

De los problemas a resolver por estas organizaciones el $18,5 \%$ se relacionan con la presión en el trabajo, el $7 \%$ con discriminación y el $6 \%$ por acoso sexual.

El $17 \%$ de estas organizaciones realiza la planeación de los recursos humanos mediante la evaluación de los recursos actuales, el $9 \%$ por la evaluación de pruebas futuras, el $11 \%$ teniendo en cuenta el desarrollo de un programa para satisfacer las necesidades futuras y el $14 \%$ por la proyección de la demanda de productos y servicios.

El 36\% no realiza los análisis de puestos, el resto de las organizaciones que lo hacen se valen de la observación directa, los cuestionarios, las entrevistas y los métodos combinados.

El $36 \%$ no realiza evaluación del desempeño y el $62 \%$ no dispone de un sitio web para reclutar personal en línea. El $33 \%$ de los trabajadores se capacitan, de ellos solo el $8 \%$ están certificados.

El éxito de cualquier emprendimiento depende, principalmente, de la flexibilidad y de la capacidad de innovación que tengan las personas que integran la organización.

La tecnología y la información están al alcance de todas las organizaciones, por lo que la ventaja competitiva que puede diferenciar a unas de otras radica en la capacidad que tengan las personas que las integran para adaptarse al cambio.

\section{Conclusiones y Recomendaciones}

1) La caracterización realizada en las micro, pequeños y medianos empresas del clúster de cuero y calzado de la Provincia de Tungurahua, permitió conocer sus principales fortalezas y debilidades y posibles áreas de mejora.

2) La integración realizada de la actividad en los negocios de cuero y calzado a las áreas de enfoque del capital intelectual, permitió identificar los elementos que tienen posibilidades de generar valor para la organización.

3) La información recopilada permite sentar las bases para diseñar una metodología para la gestión del capital intelectual en el clúster objeto de análisis.

4) Fortalecer la agremiación del clúster cuero y calzado de la provincia de Tungurahua para lograr la competitividad.

5) Incluir en la metodología, estrategias que permitan potenciar la internacionalización de los productos del clúster.

\section{Referencias Bibliográficas}

Aceves, J. R. (2010). Modelo de evaluación del capital intelectual en las actividades logísticas en empresas manufactureras (Inf. Téc.). México DF.
Alama, E. M. (2008). Capital intelectual y resultados empresariales en las empresas de servicios profesionales de España (Inf. Téc.). Madrid.

Alonso, C. (2014). Orientaciones para implementar una gestión basada en procesos. Ingeniería Industrial, Vol. XXXV/No. 2, 159-171.

Betancur, C. M. (2015). Una reflexión sobre la relación existente entre el capital (Inf. Téc.). Medellín.

Borras, F. (2014). Los activos intangibles: factores de éxito de las empresas cubanas. Cofin Habana, 47-56.

Borrás, F., Ruso, F., y Campos, L. (2011). Bases conceptuales para un modelo cubano de valoración e información contable del capital. Economía Autónoma de la Universidad Autónoma, 103-130.

C. G. J., Salazar-Mesa, L., R.-J. (2013). Indicadores de capital intelectual. Memorias, 11(19), 39-51.

Contreras, C., Díaz, B., y Hernández, E. (2012). Multiculturalidad: Su análisis y perspectivas a la luz de sus actores, clima y cultura organizacional prevalecientes en un mundo globalizado. Málaga: Servicios Académicos Intercontinentales S.L. B-93417426.L.

Edvinsson, L. (2007). El capital Intelectual. Barcelona: Ediciones Gestión 2000.

EUROFORUM. (1998). Modelo de Capital Intelectual "Intelect". España: La Vanguardia.

Monagas, M. (2012). El capital intelectual y la gestión del conocimiento. Ingeniería Industrial, 142-150.

Nevado, D., y López, V. R. (2002). Capital Intelectual. Valoración y Medición. Modelos, Informes, Desarrollos y Aplicaciones. Madrid: Prentice Hall.

Rivero, D. (2009). Modelo conceptual para la medición del capital intelectual y un procedimiento para su implementación. Caso hotelero (Inf. Téc.). Matanzas.

Ruso, F. (2011). Valoración y medición del capital intelectual. Cofin Habana, 32.

Simó, P., y Sallán, J. M. (2008). Capital intangible y capital intelectual:. Estudios de Economía Aplicada, 65-78.

Stewart, T. (2015). Capital Intelectual, la nueva riqueza de las organizaciones. Londres: Nicholas Brealey.

Valencia, M. (2005). El capital intelectual: capacidad competitiva empresarial. Entramado 1(1), 40-49.

Recibido: 30 de junio de 2018

Aceptado: 3 de septiembre de 2018 
